\title{
L'EDUCATION ET LA FORMATION DU PERSONNEL ENSEIGNANT DANS UN PAYS EN PLEIN DEVELOPPEMENT: LA JAMAÏQUE
}

par GILbert L. DE LANDShEeRE, Liège

Dans l'ensemble des nations qui ont conquis depuis peu leur indépendance politique ou sont sur le point de l'obtenir, la Jamaïque est un point de repère extrêmement intéressant à observer. En effet, ses réalisations éducatives peuvent être considérées comme une étape intermédiaire entre la situation des jeunes Etats d'Afrique centrale, par exemple, et celle des pays hautement industrialisés de l'Occident.

Cet article voudrait être plus qu'une synthèse de lectures et d'observations directes. Il souhaiterait rendre hommage à l'effort d'un peuple qui, sous la direction de leaders compétents, progresse à un rythme exceptionnellement rapide.

Nous avons laissé à notre exposé la forme de notes d'enquête qui convient mieux, croyons-nous, à la présentation d'un système en constante évolution.

Avant et pendant notre séjour à la Jamaïque, le Dr. Elsa $\mathrm{H}$. Walters, de l'University College of the West Indies, nous a apporté une collaboration infatigable et nous a livré le fruit de son expérience extraordinaire en matière d'enseignement dans les Caraïbes. Nous avons aussi trouvé partout une aide agissante au Ministère de l'Education, à Kingston, et avons pu notamment disposer du rapport du Committee on the Development of Teacher-Training in Jamaica, document dont nous utilisons de nombreuses données. Enfin, Mr. J. J. Figueroa et ses collègues du Département de l'Education de l'Université des Indes Occidentales nous ont réservé le meilleur accueil.

Il n'est pas possible de traiter de la formation des maîtres d'un pays déterminé sans se référer à la situation générale de celui-ci et au degré de développement de son système éducatif.

La première partie de cette étude présente donc quelques traits de l'arrière-plan nécessaire.

La seconde partie est entièrement consacrée à la formation et au perfectionnement des maîtres. Le problème du développement de l'enseignement élémentaire dominant tous les autres, nous nous sommes surtout arrêté à la préparation des instituteurs. 


\section{Le cadre}

Ile la plus importante de la nouvelle Fédération des Indes Occidentales (fondée en 1958), la Jamaique en constitue aussi le centre culturel.1) Longue de $240 \mathrm{~km}$ et large de $80 \mathrm{~km}$, elle couvre environ $11.500 \mathrm{~km}^{2}$ et compte 1,7 million d'habitants dont 1/5 résident à Kingston, la capitale. La population comprend $98 \%$ de gens de couleur, dont une forte majorité de noirs.

Découverte par Christophe Colomb en 1494 et donc espagnole à l'origine, la Jamaïque devint colonie anglaise en 1655. Cette année-ci elle obtiendra son indépendance mais restera membre du Commonwealth britannique. Aujourd'hui elle jouit déjà d'une autonomie à peu près aussi complète que le Canada.

La langue véhiculaire est l'anglais. Toutefois, celui-ci est fort dénaturé dans les milieux non-cultivés: grammaire simplifiée, mots omis, consonnes finales supprimées, intonation différente.

Les ressources principales de l'île sont, dans l'ordre: la bauxite, la canne à sucre, le tourisme, les bananes, le rhum et le café. Le pays fait un gros effort d'industrialisation; l'implantation d'entreprises industrielles nouvelles et la construction d'hôtels sont encouragées par une exemption de tous impôts pendant 7 ans.

La religion anglicane est la plus répandue; on trouve ensuite des baptistes, des méthodistes, des presbytériens, des catholiques romains et des juifs.

Bien qu'elle fut découverte par l'Occident il y aura bientôt cinq siècles et malgré des échanges culturels déjà anciens avec l'Angleterre, la Jamaïque est encore un pays jeune, en plein développement.

Parmi les impressions qui assaillent le visiteur, deux dominent: la volonté des forces vives de la nation de contribuer à la grandeur d'un pays où le souvenir de l'esclavage reste vif, et la compétence et la maturité des dirigeants - qui continuent d'ailleurs de s'entourer de spécialistes anglosaxons partout où ils en sentent le besoin.

La Jamaïque est loin d'être un pays sans problèmes. Elle se débat contre de grosses difficultés économiques (p. ex.: nécessité urgente de relever le revenu per capita), sociales (p. ex.: bidonvilles, surpopulation de certaines zones) et politiques (p. ex.: les Rastafari, secte anarchiste de plus en plus puissante).

Devant pratiquer une politique d'urgence dans de nombreux domaines,

1) La Fédération des Indes Occidentales compte 10 membres: la Jamaĩque, la Barbade, la Trinité et Tobago, Montserrat, Antigua, la Dominique, Grenade, Saint Kitts-Nevis-Anguila, Sainte-Lucie, et Saint-Vincent. 
le Gouvernement a néanmoins estimé que les efforts principaux devaient être consacrés à un programme à long terme et a, pour cette raison, réservé la priorité à l'éducation.

Cette volonté apparaît nettement dans les budgets 1958-1959 et 19601961 dont nous reproduisons les postes principaux:

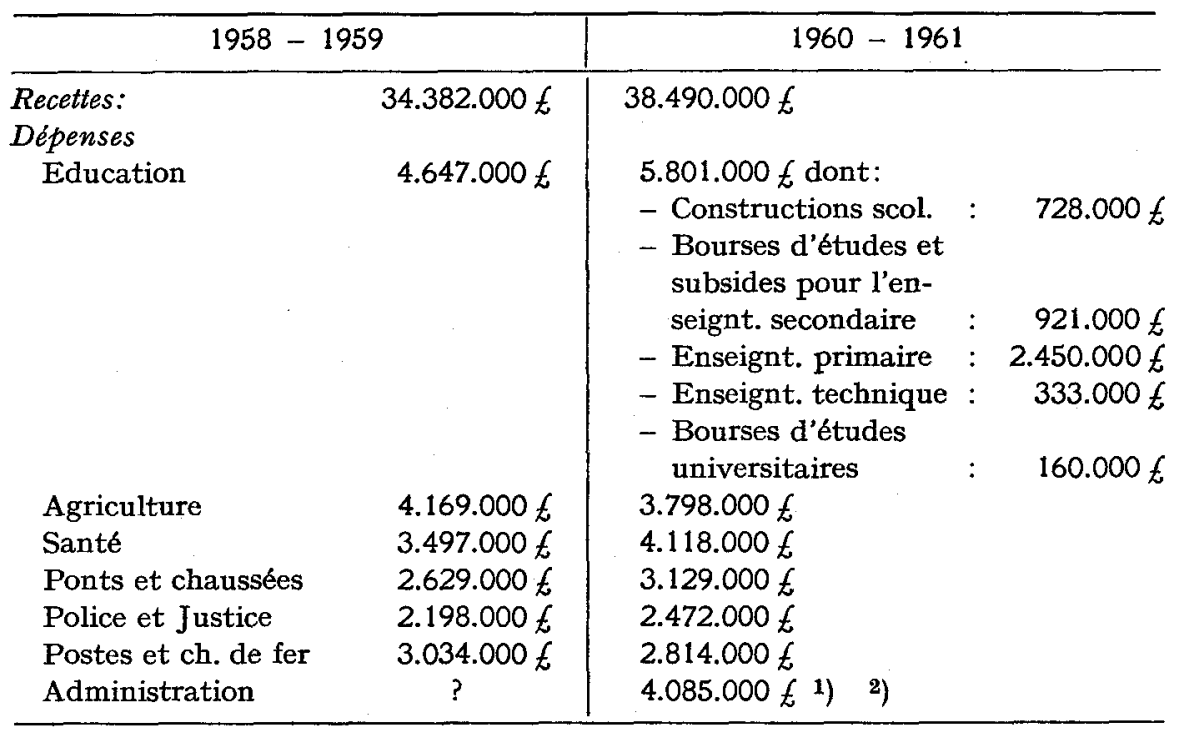

Notons enfin que la première institution commune aux dix pays de la Fédération des Indes Occidentales fut l'University College of the West Indies dont la fondation précéda de dix ans la constitution politique de la Fédération.

L'Université joue un rôle primordial dans le développement de la nouvelle nation. Dans le domaine de l'éducation, notamment, on ne peut qu'admirer l'esprit de saine collaboration qui règne entre les services gouvernementaux, les anciennes institutions d'enseignement et le nouveau département de pédagogie de l'Université.

Lorsqu'en 1954, le Dr. E. Walters quitta sa chaire de pédagogie à l'Université de Londres pour aider la Fédération à développer son enseignement, elle trouva en maints endroits une situation qui fut, par

1) Cf. Jamaica Now, juillet 1958, no 1, Vol. 1 et avril 1960, no 10, Vol. 2.

2) A titre comparatif, signalons qu'en 1951, au moment où 1'UNESCO fit son enquête mondiale sur l'éducation, la Jamaïque consacrait proportionnellement 2,5 fois plus d'argent à l'éducation que le Congo Belge alors que, dans ce dernier pays, le taux d'analphabétisme était estimé à $66 \%$ contre $26 \%$ pour l'île des Caraibes.

cf. UNESCO, L'éducation dans le monde, Paris, 1955, p. 133 et p. 758. 
après, décrite par le Carnegie Corporation of New York Quaterly ${ }^{1}$ ): cadres de l'enseignement élémentaires squelettiques, plus de la moitié des maîtres non qualifiés, "élèves-instituteurs" commençant à enseigner dès 14 ans - des classes comptant parfois plus de 70 élèves, bâtiments insuffisants, matériel scolaire rudimentaire. Les méthodes d'enseignement souffraient aussi de ces conditions: l'apprentissage de mémoire et la récitation occupaient la plupart du temps.

Certes, la situation n'était plus aussi grave à la Jamaïque même, sauf en quelques points isolés. Elle était cependant loin d'être brillante. C'est pourquoi les premiers efforts concertés portèrent sur la formation des maîtres.

Mais, avant d'étudier cette question, il est nécessaire de jeter un regard sur le système éducatif général, tel qu'il se présente aujourd'hui.

La Jamaĩque a peu développé l'enseignement maternel. Les autorités nous ont paru conscientes de l'importance de cette première éducation scolaire, 2) mais comme dans beaucoup de pays en voie de développement, il leur a été impossible de généraliser en même temps le gardien et le primaire.

Toutes les écoles élémentaires urbaines ou rurales que nous avons visitées sont surpeuplées. Un exemple parmi d'autres: l'école rurale de Sanguinetti compte 395 élèves pour 4 instituteurs (dont deux seulement ont reçu une formation pédagogique satisfaisante) et un directeur.

Le plus souvent, l'enseignement se donne dans des conditions que nous considérerions comme inacceptables. A cause de la grande chaleur et faute de pouvoir utiliser les procédés modernes de conditionnement, beaucoup d'écoles ont été construites en préaux. Il n'est donc pas rare de trouver 4 ou 5 classes, de 60-70 élèves chacune, que pratiquement rien ne sépare pendant les leçons.

Or, malgré les nombreuses sources d'inattention et d'indiscipline, le bruit est relativement peu élevé et, si le déchet est grand, la qualité de certains résultats obtenus surprend.

Le silence des enfants s'expliquerait en premier lieu par l'attitude parentale: dans les familles, les enfants sont en effet constamment invités à garder le silence en présence des adultes; en outre, la motivation serait très forte, la jeunesse jamaïcaine s'entendant répéter que seules les études la sauveront de la pauvreté. Il conviendrait d'examiner si d'autres fac-

1) Cf. University in the Sun, juillet 1960, Vol. VIII, $n^{\circ} 3$.

2) Nous avons d'ailleurs pu visiter, entre autres, le jardin d'enfants de Central $B r a n c h$, à Kingston, où malgré la surpopulation on faisait de l'excellent travail. 
Organisation de l'enseignement

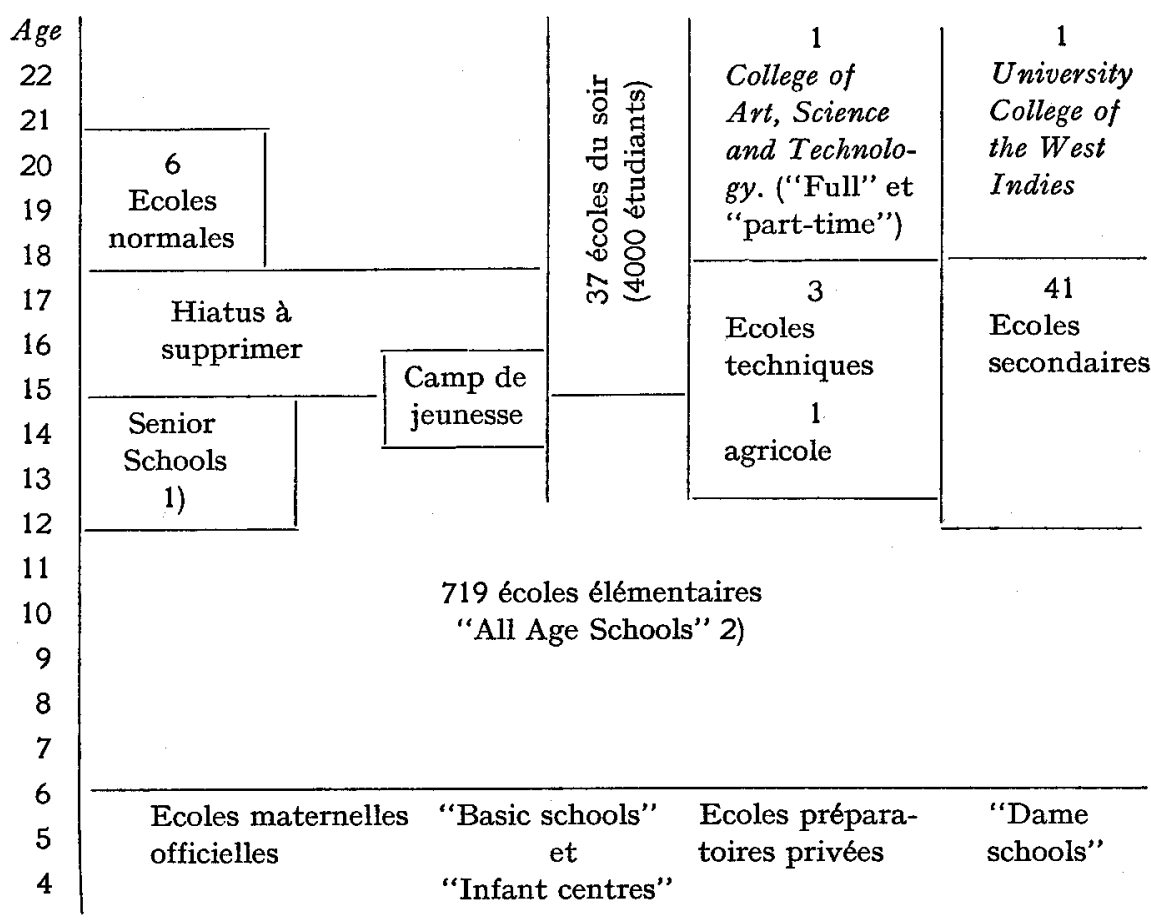

1) Les "Senior Schools", écoles du secondaire inférieur sont relativement peu nombreuses. Elles sont plus proches des "Comprehensive Junior High Schools" américaines que des écoles moyennes belges: l'aspect pré-professionnel, pré-technique ou ménager y est bien développé. La Papine School, près de Kingston, constitue un très bel exemple de ce type d'école.

2) Les "All Age Schools" sont essentiellement des écoles élémentaires qui dispensent l'enseignement jusqu'à 15 ans.

teurs physiques, psychologiques et culturels n'influencent pas les élèves, mais nous n'en avons pas eu le loisir.

Il est difficile de porter un jugement général sur la valeur de l'enseignement primaire, les leçons auxquelles nous avons assisté étant de valeur fort différente. Les meilleurs maîtres nous semblent atteindre le niveau enviable où l'enseignant se dégage du formalisme; chez les autres, on trouve encore beaucoup de leçons mécaniques et même de mémorisation brutale.

Mais les autorités pédagogiques qui guident les instituteurs et les institutrices semblent avoir compris où se trouvent les vrais problèmes et agissent en conséquence. C'est sans doute pourquoi l'enseignement jamaicain donne une impression de dynamisme. On sent souvent un effort de concrétisation et l'usage des audio-visuels est encouragé avec succès. 
Une grande importance est accordée à la diététique. Non seulement des repas bien équilibrés sont servis à midi (collaboration de l'UNICEF) mais, dans plusieurs classes, nous avons trouvé des tableaux fort intuitifs, souvent élaborés par les instituteurs mêmes, soulignant la nécessité et les moyens d'une alimentation rationelle. Les informations recueillies au Ministère de l'Education confirment d'ailleurs le sentiment de l'observateur: la jeunesse jamaïcaine est saine.

Les maîtres sont aidés de façon de plus en plus efficace par des instructions précises, sans être entravantes. Ainsi, le syllabus qui accompagne les nouveaux livres d'apprentissage de la lecture, édités par le Ministère, apporte certainement une aide précieuse. Il faut aussi noter deux excellents petits ouvrages rédigés par des commissions pédagogiques: Suggestions to Teachers in Primary Schools (1959) et Suggestions to Teachers in Senior Schools and Departments (1961).

Le temps nous a manqué pour visiter l'école d'agriculture et les établissements du secondaire supérieur, généraux et techniques; ces derniers ne sont d'ailleurs encore fréquentés que par une minorité.

Nous ne nous arrêterons pas non plus à l'University College of the West Indies (UCWI). L'Université comporte les facultés et écoles suivantes: arts, sciences naturelles, sciences sociales, médecine, éducation et une école d'agronomie créée récemment à la Trinidad. Comme les autres, le département de l'éducation est en plein développement.

Nous ne pouvons terminer ces notes schématiques sans souligner l'encouragement accordé par le gouvernement à tous les mouvements et aux clubs de jeunesse. Il existe actuellement 198 clubs réunissant au total 7.519 membres.

Nous avons pu assister à la séance annuelle statutaire du Conseil des Clubs de Jeunesse de la Jamaïque. Cette séance était présidée par le Gouverneur de l'île et nous avons été impressionné par l'intérêt agissant des diverses autorités présentes.

Enfin, nous nous sommes rendu au camp de jeunesse de Cobbla, situé dans la montagne. Ce camp accueille des garçons de 14 à 15-16 ans qui ne fréquentent plus d'école et dont l'oisiveté constituerait un grave danger moral. Les installations présentent un certain aspect militaire. Mais nous avons été frappé par l'atmosphère détendue qui régnait dans ce groupe de 650 jeunes gens. Ils font vivre la communauté par leur travail et peuvent apprendre différents métiers: tailleur, mécanicien de garage, coiffeur, agriculteur, horticulteur, éleveur (petit et grand élevage), etc.... 


\section{La Formation des maîtres}

La Jamaĩque éprouve un urgent besoin de maîtres plus nombreux et mieux formés.

D'une part, le nombre d'élèves augmente rapidement, non seulement à cause de l'accroissement de la population, mais aussi par suite de la politique gouvernementale qui, depuis 1957, s'est fixé pour objectif d'assurer au moins 5 années d'éducation élémentaire à tous les enfants de l'île qui veulent s'instruire. Il n'existe pas encore de scolarité obligatoire, mais elle est envisagée pour un avenir assez proche.

D'autre part, un grand nombre d'instituteurs en fonction n'ont reçu aucune formation pédagogique systématique. L'objectif actuel est que les $2 / 3$ des instituteurs en fonction soient formés pour 1967.

Cet effort est conforme aux résolutions de la Première Conférence Régionale sur la Formation des Maîtres, tenue en 1957, à la Trinidad.1) Cette ambition semble modeste, mais se heurte en réalité à de grandes difficultés, d'ordre budgétaire notamment: plusieurs îles de la Fédération ne pourraient pas payer les traitements plus élevés auxquels auraient droit les enseignants diplômés.

Il existe 6 écoles normales à la Jamaĩque:

4 sont du type classique européen;

1 est un centre de formation pour les meilleurs maîtres qui professent sans avoir jamais fréquenté d'école de pédagogie;

1 est un centre de formation accélérée en 22 semaines (cours d'orientation).

Les quatre premières écoles sont anciennes: l'aînée, Mico College, fut fondée en 1836, soit trois ans après l'abolition de l'esclavage; Bethlehem College, Shortwood College et St Joseph's College datent respectivement de 1861,1885 et 1887 .

Les centres de formation sont, par contre, récents; leur fondation coïncide avec l'acheminement du pays vers son indépendance: 1956 (Moneague Training College) et 1958 (Caledonia Junior College).

Le développement des quatre premières écoles fut relativement lent.

1) A cette époque, les pourcentages de maîtres en fonction ayant reçu une formation pédagogique systématique dans une Ecole Normale ou dans un Training College étaient estimés comme suit:

$\begin{array}{lrlr}\text { Antigua } & 40 \% & \text { Montserrat } & 21 \% \\ \text { La Barbade } & 25 \% & \text { Saint Kitts } & 20 \% \\ \text { La Dominique } & 9 \% & \text { Sainte-Lucie } & 6 \% \\ \text { Grenade } & 8 \% & \text { Saint-Vincent } & 6 \% \\ \text { La Jamaique } & 44 \% & \text { La Trinite } & 45 \%\end{array}$

Cf. Elsa H. Walters, Teacher Training Colleges in the West Indies, London: Oxford University Press 1960, p. 123. 
Mico College, le plus important, ne comptait encore que 50 élèves en 1886. Or, il fournissait, et fournit encore parfois, des instituteurs pour toutes les Caraïbes. Beaucoup de leaders actuels sont des anciens élèves de Mico.

Les progrès ne s'accélérèrent vraiment qu'après la Seconde Guerre mondiale et, actuellement, les populations scolaires sont approximativement les suivantes ${ }^{1}$ ):

$\begin{array}{lll}\text { Mico College } & 200 \text { étudiants } & \\ \text { Bethlehem College } & 120 & \text { (60 en 1946) } \\ \text { Shortwood College } & 200 & \text { (60 en 1952) } \\ \text { St Joseph Training College } & 120 & \end{array}$

Le petit nombre d'élèves diplômés dans le passé et le fait qu'ils ne provenaient pas tous de la Jamaïque expliquent la situation difficile d'aujourd'hui.

En 1955, on estimait que $56 \%$ des 4.500 enseignants en service n'avaient reçu aucune formation pédagogique spécialisée. La qualité des autres membres du personnel était naturellement fort variable. La même année, un programme d'intensification de l'éducation fut élaboré et mis en application avec les conseils éclairés de quelques spécialistes britanniques de la formation des maîtres, dont l'éminente Miss E. Walters. Un an après, le Moneague College entrait en activité et il a, depuis lors, perfectionné 100 enseignants chaque année. Depuis 1958, à raison de deux sessions par an, le Caledonia Junior College a, de son côté, entraîné un millier d'étudiants.

Comme dans les notes qui suivent, nous faisons allusion à diverses catégories administratives de maîtres, nous croyons utile de présenter un tableau de synthèse de la structure du personnel enseignant. ${ }^{1}$ )

\section{Classification du personnel enseignant}

\section{Enseignement primaire}

Directeurs d'école: I Ecole de plus de 500 élèves

$\begin{aligned} \text { II } & \text { de } 300 \text { à } 500 \\ \text { III } & \text { de } 150 \text { à } 300 \\ \text { IV } & \text { de moins de } 150 \text { élèves }\end{aligned}$

1) Ces chiffres ainsi que de nombreuses autres données qui suivent sont extraits $\mathrm{du}$ Report of the Committee on the Development of Teacher Training in Jamaica, Ministry of Education, Jamaica, W.I., déc. 1960. 
Instituteurs diplómés d'Ecole normale:

Catégorie I Diplôme d'Ecole normale + Cambridge Higher School Certificate.

II Diplôme d'Ecole normale, uniquement.

Instituteurs non diplómés d'Ecole normale:

Catégorie I Pas de formation pédagogique, mais Higher School Certificate.

II Ont passé le 2ème examen de l'Ecole normale ou possèdent le School Certificate, Grade $I$ or 2.

III Ont passé le ler examen de l'Ecole normale ou possèdent le School Certificate, Grade 3.

IV Possèdent seulement le Jamaica Certificate of Education ou son équivalent.

Engagés à l'essai; disposent de 6 ans pour acquérir les titres requis en catégorie III. Si, au bout de ce délai, ils n'ont pas subi avec succès les épreuves requises, ils sont rayés de la liste des instituteurs qui peuvent fonctionner dans les écoles subsidiées par l'Etat.

Maîtres spéciaux (375) : économie domestique, travail manuel, enseignement artistique, agriculture.

\section{Enseignement secondaire}

41 High Schools (Grammar Schools) subsidiées, comptant 843 enseignants:

479 détiennent un diplôme universitaire;

364 se rangent dans les catégories allant de: Instituteur diplômé catégorie I à non diplômé, catégorie III.

Pour mémoire: 3 écoles techniques, 2 centres de formation pratique, 1 école d'agriculture, 6 écoles normales.

\section{La Situation jusqu'en 1957}

Jusqu'en 1957, il suffisait d'avoir terminé l'école primaire et d'avoir passé un petit examen (Pupil Teachers' Examination) pour être autorisé à enseigner.

Après quelques années de pratique, les plus ambitieux ou les plus favorisés de ces jeunes "élèves-maîtres" suivaient les cours d'une école normale ( 3 ans) ou se présentaient aux trois examens successifs après une préparation autodidacte.

Le plus grand nombre restait fort inculte.

\section{Après 1957}

Comme nous l'avons dit, 1957 vit le début d'un effort considérable, tant sur le plan quantitatif que sur le plan qualitatif.

La formation des maîtres selon la voie normale (école élémentaire, éventuellement un peu d'études secondaires, puis école normale de 3 ans) ne sera pas étudiée ici. C'est l'acheminement traditionnel. 
Nous nous arrêterons, par contre, aux cours de formation accélérée et aux cours de perfectionnement.

\section{Formation accélérée}

A l'heure actuelle, et sauf quelques exceptions, on ne permet plus que de jeunes adolescents commencent à enseigner dès leur sortie de l'école élémentaire. Ils doivent, en principe, avoir atteint l'âge de 18 ans pour devenir instituteurs. Parmi ceux qui se destinent à cette carrière, les uns restent oisifs en attendant l'âge requis, ${ }^{1}$ ) les autres fréquentent une ou deux années de l'école secondaire.

Avant d'entrer en fonction, les candidats doivent subir le Third Jamaican Local Examination (anciennement: Pupil Teachers' Examination), petite épreuve d'aptitude servant à éliminer les jeunes gens insuffisants sur le plan intellectuel, moral ou physique.

Les jeunes maîtres acquièrent leur première expérience de façon tout à fait empirique. Comme nous l'avons signalé dans le tableau, p. 48-49, ils disposent de 6 ans pour passer un examen équivalent à celui de la première année d'école normale.

Toutefois, d'immenses progrès sont maintenant réalisés grâce aux cours de formation accélérée de 22 semaines, donnés au Caledonia Junior College. Après quelques tâtonnements, l'école de Caledonia est devenue un internat. ${ }^{2}$ ) Les locaux, tant de travail que de séjour, sont loin d'être luxueux. Il s'agit d'une ancienne maison privée, assez spacieuse, dont les moindres recoins sont exploités. Le staff est peu nombreux: 5 professeurs et un directeur. On livre ici une véritable bataille contre le temps et les difficultés et l'admiration du visiteur va également à l'opiniâtreté et au dévouement total des maîtres et à la bonne volonté des élèves.

Le succès d'une entreprise comme celle-ci dépend, dans une large mesure, de son chef. Le directeur actuel, issu de l'enseignement primaire, dirige son école de main de maître. L'administration centrale lui accorde une indépendance presque totale; il est seul à décider, en dernier ressort, de l'admission d'un élève, ce qui lui a permis, dès le début, de dresser un barrage inflexible contre tout favoritisme politique. Une liberté tout aussi grande lui est accordée quant à l'utilisation du budget.

Quand la confiance accordée est méritée - ce qui est le cas - une telle politique est évidemment la plus efficace. Cet esprit de pionniers où le

1) Voir le plan d'organisation, p. 45: "Hiatus."

2) Le Caledonia Junior. College est situé au centre de Kingston. Les étudiants, en majorité des jeunes filles, y viennent de toutes les parties de l'île. Non seulement les problèmes de logement et d'entretien étaient difficiles à résoudre individuellement, mais les dangers moraux étaient grands. De plus, la vie d'internat permet un entraînement plus intensif. 
sens des responsabilités se combine à la volonté d'action explique certainement dans une large mesure les progrès rapides que nous avons souvent constatés à la Jamaïque.

Deux promotions de 150 élèves chacune sortent chaque année de Caledonia.

La formation accélérée poursuit trois buts fondamentaux: perfectionner, autant que possible, la connaissance de la langue maternelle; fixer fermement chez l'élève les notions d'anglais et d'arithmétique qu'il devra enseigner; faire acquérir, surtout par la pratique et l'observation dirigée, sur le terrain, les premiers éléments de psychologie pédagogique et de psychologie. Les étudiants apprennent aussi à utiliser et à confectionner le matériel didactique de base.

L'éventail des cours est le suivant:

\section{A. Branches obligatoires}

1. Education: psychologie méthodologie pratique de l'enseignement

2. Anglais: grammaire, littérature générale, littérature enfantine, critique de livres, etc. ...

3. Principes d'arithmétique.

4. Travail individuel: enquête sur un aspect de la communauté ou construction d'un matériel didactique.

5. Arts.

\section{B. Branches à option}
Groupe I: éducation physique travail à l'aiguille
Groupe II : étude du milieu religion.

N.B.: Le choix doit obligatoirement se porter sur une branche de chaque groupe.

Examens. - Les questions sont rédigées par les professeurs et soumises pour approbation à un professeur du Département de l'Education de l'Université.

Tout académisme est évité. La formation se veut utilitaire et immédiatement efficace: pensée et action sont étroitement associées.

Nous avons, entre autres, visité la classe d'une élève récemment sortie de Caledonia, à Denham Town. L'institutrice donnait une leçon de grammaire, assez délicate pour une débutante. Il était évident qu'elle suivait 
fidèlement un plan de leçon type. Malgré des maladresses inévitables, le but de la leçon fut atteint. On ne peut guère en demander plus.

Jusqu'à présent, le Caledonia Junior College a travaillé dans des conditions très difficiles. On espère que le rapport professeur/élèves sera bientôt porté à $1 / 15$ et qu'un sous-directeur sera nommé. On envisage aussi la création d'une seconde école du même type en un autre endroit de l'île.

\section{Cours par correspondance}

Nous avons dit plus haut que les jeunes gens entraînés à Caledonia diposent de six ans pour présenter la première épreuve de l'enseignement normal régulier. Pour les aider à se préparer, le Gouvernement organise un cours par correspondance qui, de nouveau, vise à perfectionner les connaissances générales et la formation pédagogique.

Le programme d'études comprend l'anglais, l'arithmétique, la pédagogie et une ou deux branches à option (mathématiques, histoire, géographie, littérature anglaise, religion). L'élève devra aussi lire un certain nombre d'ouvrages mis à sa disposition. Il devra en outre soumettre des préparations de leçons qui seront renvoyées après correction. Si le jeune maître a la chance de travailler aux côtés d'un instituteur expérimenté, il trouvera en ce dernier un conseiller fort utile.

Il n'y a pas de délai rigide pour suivre le cours. Les organisateurs ont voulu tenir compte de la grande variété des conditions dans lesquelles les élèves travaillent. Normalement, le cours est couvert en deux ou trois ans et est suivi d'un examen écrit. Si l'épreuve est réussie, le maître passe de la catégorie IV à la catégorie III, son traitement est amélioré et son emploi peut devenir définitif. De plus, l'élève-maître peut alors s'inscrire à un cours normal de 2 ans qui lui procurera le titre d'instituteur diplômé. Fin 1960, 453 étudiants suivaient les cours par correspondance et fin 1961 , les premiers candidats se présenteront à l'examen.

La Commission pour le Développement de la Formation des Maîtres à la Jamaïque recommande actuellement une extension de ces cours. Elle souhaite que la direction en soit assurée par un spécialiste de l'enseignement par correspondance. Elle souligne, en outre, que les cours doivent conduire l'étudiant à l'observation directe et au travail pratique et qu'il faut prendre toutes les précautions possibles pour empêcher ou décourager l'étude mécanique ne visant qu'au succès de l'examen.

La technique de cet examen n'est d'ailleurs pas encore définie et on espère trouver un moyen qui réduirait la composition écrite au minimum.

Il est aussi important d'établir des relations humaines aussi étroites que possible entre l'étudiant et le centre du cours par correspondance. 
Dans la pratique, beaucoup d'élèves rendent visite au directeur du cours à l'occasion d'un passage à Kingston; souvent, les entretiens se transforment en véritables leçons particulières.

Nous pensons toutefois que ce n'est pas suffisant et que les visites au centre ne devraient pas être laissées au seul hasard. Des séminaires devraient, à notre avis, compléter le cours par correspondance.

\section{Cours de perfectionnement}

Le Moneague Training College s'adresse aux maîtres qui fonctionnent depuis plusieurs années déjà et n'ont souvent suivi aucun cours systématique de pédagogie. La formation à Moneague dure un an. Chaque promotion compte une centaine de participants (des institutrices surtout bien que, théoriquement l'institut soit mixte) qui, pendant leurs études, continuent à percevoir leur traitement normal mais doivent payer leur pension (environ les $2 / 3$ du traitement). Selon le degré de réussite, les diplômés de Moneague voient leur statut s'améliorer, parfois dans une mesure considérable.

Pour être admis à suivre ces cours, les candidats doivent introduire une demande personnelle, appuyée par des certificats du directeur de l'école où ils travaillent, de l'inspecteur et d'un prêtre ou d'un pasteur; ils doivent aussi se présenter à une interview. On ne retient, en général, que les candidats âgés d'au moins 24 ans qui, de façon autodidacte, ont acquis des connaissances correspondant au programme de la première année d'école normale.

Le staff de l'école comprend: une directrice, 8 professeurs (pédagogie, méthodologie, anglais, sciences, économie domestique, arts et travaux manuels, musique), 1 bibliothécaire chargé de cours, une économe et une assistante, une secrétaire et une dactylo.

Selon leur désir, les étudiants sont préparés soit pour l'enseignement primaire (Junior Schools, 6-12 ans), soit pour l'enseignement secondaire inférieur (Senior Schools, 12-15 ans).

Les cours suivants sont offerts:

\section{Formation professionnelle}

Etude de l'enfant, psychologie. Principes de pédagogie.

Méthodologies générale et spéciales.

Anglais, y compris littérature.

\section{Formation théorique}

Groupe A: sciences et histoire

Groupe B: géographie et mathématiques

Groupe $\mathrm{C}$ : musique et religion. 


\section{Formation pratique}

Economie domestique

Arts et travail manuel

Education physique.

Tous les élèves doivent suivre les cours de la catégorie I, plus le chant choral et les sports (jeux).

Les maîtres qui se spécialisent pour l'enseignement primaire doivent, en outre, choisir 3 branches (une de chaque groupe) de la catégorie II et une branche de la catégorie III.

Ceux qui se destinent à l'enseignement secondaire doivent pratiquement suivre tous les cours.

Les élèves doivent, de plus, enseigner au moins 5 semaines à l'école d'application et soumettre, en fin d'études, un travail personnel, sur un sujet au choix, comptant 5.000 mots minimum.

Examens. - Les branches de la catégoie I font l'objet d'un examen écrit d'un niveau élevé, approximativement celui de la classe de poésie des lycées belges. Pour les catégories II et III, l'évaluation se fait pendant toute l'année. En cas d'échec à l'examen écrit, dans le travail personnel ou en pratique de l'enseignement, le candidat peut se représenter, deux fois maximum, à des sessions suivantes, à condition de se soumettre chaque fois à une nouvelle période d'entraînement.

Le programme des activités et l'atmosphère de l'école montrent que l'on s'efforce non seulement d'accroître la compétence professionnelle des enseignants, mais que l'on vise aussi à l'épanouissement de toute leur personnalité. Des clubs et des associations d'étudiants, des concerts, des conférences sont particulièrement précieux pour des maîtres ruraux qui vivent d'habitude dans l'isolement.

\section{Le Conseil responsable de la formation des maîtres (Board of Teacher-Training)}

Ce conseil fonctionne depuis 1956 et se compose des directeurs et directrices des écoles normales, d'un délégué du Ministre de l'Education et d'un représentant de l'Université.

Le conseil est responsable devant le Ministre et a pour mission:

a) de donner avis sur tous les problèmes éducatifs concernant la formation des maîtres, y compris les programmes d'enseignement; $b$ ) d'organiser les examens et d'en superviser la correction.

Il n'est pas indiqué de décrire ici, par le menu, toutes les activités du Conseil. En voici seulement quelques aspects frappants. 
Approbation de nouveaux programmes de cours. - Tout changement de programme est d'abord discuté, dans l'école qui prend l'initiative du projet, entre les professeurs de la branche et le directeur. Le projet est ensuite soumis à une commission de spécialistes composée de tous les professeurs de la branche intéressée, fonctionnant dans l'enseignement normal, et présidée par un professeur de l'Univer sité. De ce fait, l'Université a donc l'occasion d'exercer une influence directe sur les programmes.

Le Conseil intervient à son tour et envisage surtout l'incidence de la modification proposée sur l'ensemble du programme.

Est-il besoin de souligner l'importance du processus démocratique qui associe tous les maitres aux efforts de perfectionnement des études?

Examens. - Pour chaque branche, les questions d'examens sont préparées par la commission de spécialistes que nous venons de décrire. Ici aussi, le contact est donc direct avec l'Université et le rapport entre les professeurs des différentes écoles est assuré. Il nous semble remarquable que les correcteurs des examens attirent immédiatement l'attention du Conseil s'ils rencontrent des réponses stéréotypées tendant à indiquer qu'une école se soucie plus du succès aux examens que de l'éducation véritable.

Dans son rapport récent, le Committee on the Development of TeacherTraining in Jamaica recommande une réorganisation du Conseil qui comprendrait alors 18 membres:

3 représentants du Ministère de l'Education, dont un serait le secrétaire du Conseil;

3 représentants de l'Université, dont deux maximum viendraient du Département de l'Education;

6 représentants des Ecoles normales: 3 directeurs et 3 professeurs;

1 représentant du College of Arts, Science and Technology;

1 représentant de l'Association des directeurs d'écoles;

1 représentant du syndicat des enseignants jamaïcains;

1 représentant du Conseil consultatif de l'Instruction publique;

1 représentant de l'Association des services de formation des maîtres;

1 citoyen dévoué au bien public, nommé par le Ministre de l'Education.

Les membres recevraient un mandat de trois ans et ne seraient qu'une fois rééligibles.

\section{Le Personnel des écoles normales}

Pour la Jamaĩque, le Committee considère que le rapport idéal professeur/étudiants serait de 1/12. Toutefois, dans les conditions actuelles, le rapport $1 / 13$ paraît seul réaliste et ce chiffre a été retenu comme norme. 
Vu le programme d'études adopté et pour assurer l'enseignement des différentes disciplines par un corps professoral employé full-time, on estime qu'une école normale devrait compter 18 professeurs et donc au moins 240 élèves. Les experts jamaïcains sont en outre d'avis que la population d'une école normale ne devrait pas dépasser 300 étudiants.

Dans le cas d'une école de 240 étudiants, les 18 professeurs se répartiraient comme suit:

4 professeurs de pédagogie

$\begin{array}{lll}4 & \quad & \text { de langue maternelle } \\ 4 & \text { pour l'histoire, la géographie et les sciences } \\ 6 & \text { pour l'économie domestique, la religion, la musique, les } \\ \text { arts et les travaux manuels, les mathématiques et l'édu- } \\ \text { cation physique. }\end{array}$

Pour un établissement comptant 300 élèves, les chiffres ci-dessus deviendraient $5-5-5-7$.

Pour chaque école (toutes des internats), on prévoit le personnel administratif suivant:

pour 240 étudiants: 1 directeur

1 comptable
1 secrétaire
2 dactylos
1 bibliothécaire
1 infirmière et 1 assistante.

Pour 300 étudiants: un sous-directeur, half-time, en plus.

\section{Perfectionnement des professeurs}

Des cours de perfectionnement, organisés selon les besoins par le Département de l'Education de l'Université sont prévus. Après quelques années de fonction, les professeurs seront aussi envoyés à l'étranger afin d'y étudier un système éducatif différent du leur et d'observer, en particulier, comment les problèmes relatifs à la formation des maîtres y sont résolus.

On se propose aussi d'envoyer tous les directeurs d'établissements dans des Universités étrangères, notamment pour y suivre des cours d'éducation comparée.

\section{Ecoles d'application}

Les différentes Ecoles normales disposent d'écoles d'application. On s'efforce de donner à celles-ci un caractère de plus en plus expérimental. Les autorités espèrent pouvoir recruter un certain nombre de professeurs 
pour les Ecoles normales dans le personnel d'élite des écoles d'application. Signalons que les Normaliens visitent le plus grand nombre d'écoles possible pendant leurs études.

Le Personnel des enseignements primaire et secondaire inférieur: les besoins

Il est du plus haut intérêt d'examiner l'estimation des besoins d'un pays en plein développement, d'autant plus que ceux-ci ont été évalués par des spécialistes expérimentés et réalistes.

Pour évaluer les besoins jusqu'en 1967, le Committee a tenu compte de six facteurs:

1. Estimation de la population enfantine du groupe d'âge de 7 à 15 ans, jusqu'en 1967.

2. Estimation du nombre d'enseignants disponibles, pour la même période, grâce notamment aux divers systèmes de formation accélérée.

3. Rapport maître/élèves réalisable, compte tenu des ressources humaines et matérielles.

4. Introduction éventuelle de la scolarité obligatoire, de 7 à 12 ans.

5. Qualification des maîtres désirés.

6. Pourcentage de maîtres formés abandonnant l'enseignement ("wastage rate"). Le reastage rate est évalué à $2 \%$, ce qui nous paraît fort peu.

Dans les conditions actuelles, il semble qu'un rapport maître/élèves de $1 / 50$ soit réaliste et qu'il le restera pendant quelques années encore. Certes, nous l'avons vu, il n'est pas rare que, dans les écoles rurales surtout, le rapport soit $1 / 75,1 / 80$. Mais on espère apporter une première correction par une meilleure répartition géographique des instituteurs.

On tient aussi compte d'environ $20 \%$ d'absences quotidiennes.

Comme il est peu probable que la scolarité obligatoire puisse être complètement appliquée pour 1967, le Committee, se référant à la fréquentation en 1959 ( $83 \%$ des enfants entre 7 et 15 ans), a considéré que $90 \%$ environ de la population enfantine du groupe d'âge considéré fréquenteraient l'école en 1967.

Ces considérations permettent d'établir les chiffres suivants:

Population enfantine

$\begin{array}{rrr}\text { Groupe d'âge de } 7 \text { à } 15 \text { ans: } & 1960 & 315.100 \\ & 1967 & 402.800 \\ 90 \% \text { de } 402.800 & & 362.520\end{array}$

On prévoit que 13.000 de ces enfants entreront dans l'enseignement secondaire proprement dit ou dans l'enseignement technique. Pour ces deux types d'enseignements, la population totale est estimée à 26.000 pour 1967. 


\section{Enseignants nécessaires}

Sur la base du rapport 1/50, les besoins seraient donc de 7.250 enseignants. Or, au ler janvier 1960, il existait: 5.137 enseignants, dont 2.537 formés et 2.600 non formés. Pour que les $2 / 3$ du personnel aient reçu une formation pour 1967, il faut porter le nombre d'instituteurs qualifiés à 4.833 . D'après la production probable des écoles normales existantes, on n'atteindrait, en 1967, que le chiffre de 3.553. Il faut donc trouver des moyens spéciaux pour préparer 1.280 maîtres supplémentaires.

Les spécialistes essaient actuellement de résoudre ce problème. Si l'on prévoit un déchet interne de $5 \%$ et un déchet externe de $2 \%$, il faut introduire chaque année 240 candidats instituteurs en plus dans les cycles de formation accélérée.

Comme le remarquent bien les membres du Committee, la situation, après 1967, sera probablement aussi compliquée étant donné le taux d'accroissement de la population toujours plus élevé.

\section{Bureau de publications}

La Jamaïque ressentait durement le manque de matériel et de manuels scolaires adaptés à sa culture. Considérant, avec raison, que la distribution de livres plus adéquats constituait un moyen facile d'élever la qualité de l'enseignement, le Ministre de l'Education créa, il y a trois ans, un Bureau des Publications.

Le travail accompli en aussi peu de temps est remarquable. Nous avons particulièrement admiré les premiers livres de lecture, composés selon les techniques américaines récentes.

Le Bureau est dirigé par deux spécialistes, assistés d'un conseiller artistique; ils disposent de deux secrétaires. Les livres sont rédigés soit par le Bureau même, soit par des enseignants qui soumettent leurs manuscrits. Si ces derniers sont acceptés, le Bureau achète les droits d'auteur. Quand les textes soumis ne répondent pas à un besoin urgent ou ne présentent pas de qualités suffisantes, le Bureau offre ses conseils mais ne se charge pas de l'édition.

Le Bureau reste en contact étroit avec les écoles où il expérimente d'ailleurs le matériel et les manuels avant de les publier. Il examine aussi les ouvrages étrangers qui lui sont envoyés et donne son avis sur l'opportunité de leur usage par les maîtres ou par les élèves.

On fait une nette distinction entre le programme de publications à court terme et le programme à long terme. Alors que, pour le premier, les besoins étaient évidents, l'élaboration du second se fera en collaboration avec l'Université. 


\section{Conclusion générale}

La Jamaïque constitue un bel exemple d'un pays en plein développement, qui prend de plus en plus conscience de ses potentialités. Il serait vain de vouloir porter un jugement absolu - d'ailleurs toujours impossible - sur la valeur de son système éducatif et de ses maîtres. Plus le travail fait dans l'île des Caraïbes nous est devenu familier, plus nous avons éprouvé de l'admiration pour la rapidité des progrès réalisés et pour le dynamisme des éducateurs et de leurs chefs.

Répétons-le, de crainte que notre enthousiasme sous-jacent n'ait parfois donné une image fausse des réalités: considérés du point de vue de nos meilleures réalisations occidentales, les standards jamaïcains restent encore souvent très bas. Toutefois, les meilleures réalisations jamaïcaines atteignent un niveau qualitatif au moins égal au nôtre.

Il sera intéressant d'examiner de nouveau la situation de ce jeune pays dans une génération.

EDUCATION AND TEACHER TRAINING

IN A RAPIDLY DEVELOPING COUNTRY: JAMAICA

by Gilbert L. De Landsheere, Liège

This article attempts to describe as objectively as possible a particular stage of educational developments and, at the same time, to pay tribute to the efforts of a country which, under skilful leadership, is making rapid strides forward. On the basis of personal observation and with the help of a wealth of material, made available to him, the author presents a sketch of the general situation and the educational system in Jamaica. He then examines the problems which have presented themselves in the training of teachers.

In 1957 , only $44 \%$ of practising teachers in Jamaica had received any systematic training. The situation was even worse in the other islands of the West Indian Federation, e.g. $6 \%$ in St. Vincent. In accordance with a resolution adopted by the First Regional Conference on Teacher Training, held in Trinidad during the same year, it is planned that, by 1967, two thirds of all teachers should have received training.

The author goes on to show how - to meet this demand - in addition to 4 ancient colleges, new institutions have been created which provide shortened and condensed forms of initial and further training. He describes the work of these new types of colleges and comments on their achievements. The important role of correspondence courses is also stressed.

Attention is drawn to the co-ordinating, directing and controlling functions of the Board of Teacher Training. There is, furthermore, a qualitative and quantitative analysis of the personnel requirements for teacher training, as well as for primary and secondary schools. Finally, the role and function of the Publications Branch of the Ministry of Education are outlined. 


\title{
SCHULWESEN UND LEHRERBILDUNG IN EINEM ENTWICKLUNGSLAND: JAMAIKA
}

\author{
von Gilbert L. DE LANDsheERE, Lüttich
}

Der Artikel versucht, eine möglichst objektive Darstellung eines Landes zu geben, das, wie der Autor besonders hervorhebt, sich unter geschickter Führung in einem äußerst schnellen Tempo entwickelt. Auf Grund persönlicher Eindrücke und einer Fülle ihm zur Verfügung gestellten Materials beschreibt Verf. die gegenwärtige Lage auf Jamaika sowie das Erziehungswesen des Landes. Er wendet sich dann besonders dem Problem der Lehrerbildung zu.

Im Jahre 1957 hatten nur 44\% aller Lehrer der Insel eine vollständige pädagogische Ausbildung genossen. Auf den anderen Inseln Westindiens war die Lage noch schlimmer; auf St. Vincent z.B. waren es nur 6\%. Auf Grund der auf der ersten regionalen Tagung über Lehrerbildung (Trinidad 1957) gefaßten Beschlüsse sollen bis 1967 zwei Drittel aller Lehrer ausgebildet sein.

Verf. beschreibt dann, wie neben den vier bestehenden Lehrerseminaren neue Ausbildungsstätten geschaffen wurden, die eine beschleunigte Aus- und Weiterbildung ermöglichen. Die Arbeit dieser neuartigen Institute wird besprochen und auf die wichtige Rolle des Korrespondenzunterrichts hingewiesen.

Eine gewisse Koordinierung, Richtunggebung und Ưberwachung liegt in den Händen des Board of Teacher-Training. Der Artikel bringt ferner qualitative und quantitative Angaben über das für die Lehrerausbildung und für den Unterricht an Volks- und höheren Schulen benötigte Lehrpersonal. Zum Schluß beschreibt Verf. die Aufgaben des Publications Branch des Erziehungsministeriums. 